\title{
Außerschulisches Lernen - ein Beitrag zur Bildung für nachhaltige Entwicklung und Inklusion
}

\section{Zusammenfassung}

Der Beitrag geht der Frage nach, inwiefern außerschulisches Lernen einen Beitrag für eine Bildung für nachhaltige Entwicklung (BNE) und eine inklusive Gesellschaft leistet. Hierzu werden Felder außerschulischen Lernens abgegrenzt und auf ihre Erfolge am Beispiel ausgewählter Best-Practice-Beispiele in Deutschland und Österreich untersucht. Schließlich werden die Analyse- und Bewertungsergebnisse hinsichtlich der Formulierung neuer Ideen zur Optimierung dieser Entwicklungen reflektiert.

Schlüsselworte: Bildung für nachhaltige Entwicklung, Inklusion, Sustainable Development Goals (SDGs), Regionales Lernen 21+, Gartenpädagogik, Berufsorientierung

\section{Abstract}

This paper follows the question how extracurricular learning/ out-of-school learning is promoting sustainable development and inclusion. In the first step, different fields of out-of-school learning will be defined. Illustrating and analyzing examples of best practice in Germany and Austria, fundamental criteria for success will be worked out. On this basis, ideas for a more effective development in this field are formulated.

Keywords: Education for sustainable development, inclusion, Sustainable Development Goals (SDGs), Regional Learning 21+, garden education, professional orientation

\section{Außerschulisches Lernen und das Potenzial für Bildung für nachhaltige Entwicklung}

Außerschulisches Lernen umfasst alle Phasen des Lernens innerhalb der Unterrichtszeit, die örtlich außerhalb des Schulgebäudes stattfinden. Dieses können kurze Lernphasen wie Unterrichtsgänge bis hin zu mehrtägigen Veranstaltungen wie der Aufenthalt in einem Schullandheim, Exkursionen oder Langzeitprojekte sein. Im engeren Sinne wird unter dem Begriff außerschulisches Lernen jedoch eine Lernform verstanden, die neben der Ortswahl weitere Qualitätskriterien erfüllt. Hier zu nennen ist in erster Linie die unmittelbare Begegnung mit dem Lerngegenstand oder Sachverhalt zur Integration in den Lernprozess (Messmer, Niederhäuser, von Rempfler \& Wilhelm, 2011, S. 7). Weitere didaktische Leitlinien sind (Diersen \& Flath, 2016a, S. 188):

- Schülerinnen- und Schülerorientierung: Einbeziehung der Schülerinnen und Schüler bei der Gestaltung des Lernprozesses.

- Handlungsorientiertes, erfahrungsorientiertes, forschendes Lernen: Gestaltung eines Lernsettings, welches auf das eigenständige Tun, das Erfahren und Erleben vor Ort und das forschende Lernen ausgerichtet ist.

- Problemorientierung: Eine problemorientierte Fragestellung steht im Mittelpunkt des Vorhabens.

- Systemorientierung: Systematische Zusammenhänge des betrachteten Mensch-Umwelt-Teilsystems, Strukturen und Prozesse werden erkennbar bzw. analysiert.

- Situationsorientierung: Die Situation vor Ort wird in den Lernprozess einbezogen, wenn unterschiedliche Phasen, Zyklen und Ereignisse das Erleben vor Ort prägen, z.B. die unterschiedlichen Phasen in der Tiermast, die Geburt eines Kalbes oder das Verladen schlachtreifer Tiere auf dem Lernort Bauernhof.

- Fachübergreifendes und fächerverbindendes Lernen: Auflösung des fachbezogenen Fokus auf den Lerngegenstand durch einen interdisziplinären Blick.

- Einbettung in den schulischen Lernprozess: Die außerschulische Lernphase wird in vor- und nachbereitende Phasen in der Schule eingebettet.

Steht es einerseits jeder Lehrperson offen, eigenständig außerschulische Lernphasen zu gestalten, indem sie frei gewählte Lernorte aufsucht, so bietet sich andererseits eine zunehmend große Anzahl außerschulischer Bildungsinstitutionen zur $\mathrm{Zu}$ - 
sammenarbeit an. Zu nennen sind hier Museen, Schülerlabore, Botanische Gärten, Zoologische Gärten, Nationalparks usw. Außerdem finden sich Anbieter, die institutionalisiert als Förderverein oder Projekt, „Lernen auf dem Bauernhof“, „Lernen im Wald“, „Lernen auf dem Acker" „Lernen in Unternehmen“ und vieles mehr anbieten. Die Zusammenarbeit mit Partnern zum außerschulischen Lernen nimmt seit Jahren stetig zu. Dass Lehrpersonen allein außerschulische Lernphasen planen und durchführen, ist demgegenüber in Summe gering und eher rückläufig einzuschätzen.

Die Zieldimension außerschulischen Lernens liegt bezüglich der Bildung für nachhaltige Entwicklung (BNE) in der Förderung von Gestaltungskompetenz (Harenberg \& de Haan, 1999). Hervorzuheben ist auch, dass beim außerschulischen Lernen die Teilnehmenden sich als selbstwirksam erleben, wenn sie merken, dass sie etwas gemeinsam vollbringen können und dass ihr Handeln für die Zukunft Bedeutung hat (Gugerli-Dolder, Traugott \& Frischknecht-Tobler, 2013).

Die Erfolge außerschulischen Lernens sind empirisch vielfältig untersucht. Aufgrund der großen Unterschiede hinsichtlich der Lernorte, Lerngruppen, dem Forschungsinteresse, dem Forschungsdesign u.v.m. sind jedoch nur wenige generalisierbare Aussagen möglich (Baar \& Schönknecht, 2018). Es lassen sich Lerneffekte herausstellen, die jedoch überwiegend für den kognitiven Bereich nachgewiesen werden. Ergebnisse $\mathrm{zu}$ den Lernprozessen an außerschulischen Lernorten und $\mathrm{zu}$ weiteren (nicht kognitiven) Kompetenzbereichen liegen bisher kaum vor. Als gesichert gilt, dass die Länge der Lernphase mit den Erfolgen korreliert und dass eine Einbindung in den Unterricht als vor- und nachbereitende Phasen positiv zu bewerten ist. Zudem ist die didaktische Gestaltung des Angebots von entscheidender Bedeutung (Baar \& Schönknecht, 2018; Dillon et al., 2006). Die inklusive Wirkung außerschulischen Lernens ist bisher kaum untersucht.

Grundsätzlich zeigen die Forschungsergebnisse jedoch große Potenziale des außerschulischen Lernens als Ergänzung des schulischen Unterrichts (Dillon et al., 2006). Es wird neben der Betonung der Bedeutung einer didaktischen Struktur und Gestaltung insbesondere die Gefahr einer unpassenden, überfrachteten (over-structured) Form diskutiert. Es ist wesentlich, die Auseinandersetzung mit dem Lerngegenstand, die reale Begegnung mit Menschen und Orten in den Mittelpunkt zu stellen. Gewählte Lernformen und Methoden sind hierauf auszurichten und Bedürfnisse, Interessen und Vorlieben der Zielgruppe zu beachten. Da Erleben und Erfahren im Mittelpunkt stehen, sollten Arbeitsmaterialien und außerschulische Lernsettings nicht die kognitive Auseinandersetzung in den Vordergrund stellen. Durch die, aufgrund der schulischen Einbettung, sichergestellte thematische Hinführung und anschließende Reflexion der außerschulischen Lehr-Lern-Einheit, findet die kognitive Ebene immer ihre Berücksichtigung.

Außerschulisches Lernen hat aber nicht nur ein großes Potenzial als Ergänzung des schulischen Unterrichts, sondern auch zur Unterstützung der Bildung für nachhaltige Entwicklung. Dabei versteht sich die nachhaltige Entwicklung als regulative Idee im Sinne Kants, was Nachhaltigkeit zu einer Orientierungsgröße für ergebnisoffene menschliche Such- und Lernprozesse macht (Enquete-Kommission „Schutz des Menschen und der Umwelt“, 1998, S. 27). Als übergeordnete Leit- linie für die Entwicklung der menschlichen Gesellschaft basiert Nachhaltigkeit auf der Vision von einer besseren, gerechteren Zukunft. Für den Weg in diese nachhaltige Zukunft werden Strategien verfolgt, die in einem diskursiven und partizipativen Prozess der Konsens- und Entscheidungsfindung immer wieder neu auszuhandeln sind. Neben der Effizienz-, Konsistenzund Permanenzstrategie gehört hierzu auch die Suffizienzstrategie, die eine Veränderung des Lebensstils hin zum bewussteren Umgang mit materiellen Gütern und der Neubewertung von Lebensqualität im Sinne von „Gut leben statt viel haben“ (Bund \& Misereor, 1996, S. 206) einfordert. Des Weiteren werden das solidarische Zusammenleben und die Prinzipien Vielfalt, Selbstorganisation, Resilienz, Lern- und Wandlungsfähigkeit sowie Fehlerfreundlichkeit vorgeschlagen (Christen, 1999, S. 58).

Der Weg zur Nachhaltigkeit führt nach Meinung der Deutschen UNESCO-Kommission (2008, S. 7) über die Bildung. Demnach ist es eine Bildungsaufgabe, den Menschen den Erwerb von Gestaltungskompetenz zu ermöglichen, damit sie fähig werden „Wissen über nachhaltige Entwicklung anwenden und Probleme nicht nachhaltiger Entwicklung erkennen zu können. Das heißt, aus Gegenwartsanalysen und Zukunftsstudien Schlussfolgerungen über ökologische, ökonomische und soziale Entwicklungen in ihrer wechselseitigen Abhängigkeit ziehen und darauf basierende Entscheidungen treffen, verstehen und individuell, gemeinschaftlich und politisch umsetzen zu können, mit denen sich nachhaltige Entwicklungsprozesse verwirklichen lassen “ (Programm Transfer-21 Koordinierungsstelle Freie Universität Berlin, 2007, S. 12). So wird die Fähigkeit, die Gesellschaft in aktiver Teilhabe im Sinne einer nachhaltigen Entwicklung gestalten zu können, ohne dies - aus pädagogischer Perspektive - zu müssen, zum zentralen pädagogischen Ziel einer Bildung für nachhaltige Entwicklung. Dass auch außerschulische Lernorte hierzu einen qualifizierten Beitrag leisten können, machen die vorhergehenden Ausführungen deutlich. So fördert ein Lernen an außerschulischen Lernorten die Fähigkeit, sich neugierig, eigenverantwortlich und handelnd Wissen anzueignen. Außerschulische Lernorte sind bedürfnisgerecht und partizipativ-gestaltbare Lern- und Erlebnisräume für alle Lernenden, in denen sie über die positive Beziehung zur Natur ein Bewusstsein entwickeln können, das auf Nachhaltigkeit und ressourcenschonendem Handeln basiert.

Vergleicht man nun konkret das Bildungspotenzial von außerschulischen Lernorten mit den pädagogischen Zielen der BNE, so zeigen die empirischen Studien von Dziewas (2008), Goller (2002), Hampl (2000), Reuschenbach (2009) und Schockemöhle (2009), dass außerschulisches Lernen die in der BNE gewünschten Kompetenzen (Künzli David \& Bertschy 2008, S. 38ff.) Anstrengungsbereitschaft, Diskursfähigkeit, Eigenaktivität, Frustrationsfähigkeit, Fehlerfreundlichkeit sowie die Fähigkeiten, analysieren, entscheiden, planen, schlussfolgern, handeln, gemeinschaftlich leben, sich austauschen und mit Komplexität sowie mit Nichtwissen umgehen zu können, fördert. Selbiges trifft auch auf die didaktischen Prinzipien der BNE (Künzli David \& Bertschy, 2008, S. 42ff.) zu. So ermöglichen und fördern außerschulische Lernorte vorausschauendes, reflektiertes, vernetztes und interdisziplinäres Denken und unterstützen die Entwicklung von Gestaltungskompetenz so- 
wie regionaler Identität (vgl. Schockemöhle, 2009 S. 283f.). Zugleich wird die Selbstständigkeit, die Fähigkeit zur Selbstorganisation, die Neugierde, die Kreativität sowie der Forscher- und Entdeckerdrang der Lernenden gefördert (Diersen \& Flath, 2016b, S. 215). Weil Schülerinnen und Schüler zunehmend mehr Zeit in Schule, Hort und anderen Einrichtungen verbringen, kommt außerschulischen Lernorten nicht nur eine Bedeutung als Lernort zu, sondern auch als Freiraum für eine gesunde physische, mentale und soziale Entwicklung. Darüber hinaus ermöglichen Lernangebote an außerschulischen Lernorten eine frühe Förderung praktischer, sozialer und methodischer Fertigkeiten und Fähigkeiten der Lernenden. Dies wird umso wichtiger, je mehr sich die Bildungslandschaft von Kindern und Jugendlichen von der realen in die virtuelle Welt verlagert. Im Berufsleben bleiben auch zukünftig solche Fertigkeiten und Fähigkeiten von Bedeutung, so dass außerschulisches Lernen die Lernenden zudem auf ihre künftige Berufstätigkeit vorbereiten kann. Dem Bereich der Berufsorientierung kommt auch beim außerschulischen Lernen eine zunehmend größere Bedeutung zu. Hier spielen insbesondere die Kontakte mit der Praxis (Wahl des Lernorts) als auch die besondere Didaktik des außerschulischen Lernens eine große Rolle.

\section{Best-Practice-Beispiele in Deutschland und Österreich UN-Dekade „Bildung für Nachhaltige Entwick- lung“ am Beispiel der Maßnahme „Regionales Lernen 21+“ und des Netzwerks „Außerschu- lische Lernstandorte BNE“ in Niedersachsen}

In Deutschland wurden im Rahmen der von der UN ausgerufenen Weltdekade „Bildung für Nachhaltige Entwicklung“ (2005-2014) Projekte und Maßnahmen ausgezeichnet, die als Best-Practice-Beispiele eingestuft werden. Eine Vielzahl der knapp 2.000 ausgezeichneten Projekte und Maßnahmen können zu dem Bereich außerschulisches Lernen gezählt werden, welches einen ersten Hinweis zur Bedeutung des außerschulischen Lernens im Feld BNE gibt (Deutsche UNESCO-Kommission e.V., o.J.). 2015 fiel der Startschuss für das UNESCO-Weltaktionsprogramm Bildung für nachhaltige Entwicklung (2015-2019). Es zielte darauf ab, langfristig eine systemische Veränderung des Bildungssystems zu bewirken und Bildung für nachhaltige Entwicklung vom Projekt in die Struktur zu bringen. Das Weltaktionsprogramm wird seit Januar 2020 im UNESCO-Programm „BNE für 2030“ fortgesetzt.

Als eine der 49 ausgezeichneten (strukturell angelegten) Maßnahmen der Weltdekade soll im Folgenden das Bildungskonzept Regionales Lernen 21+ (Abb. 1) vorgestellt werden. Die Dekade stellte über die Maßnahmen solche Ansätze heraus, die es leisten können, strukturelle Veränderungen zu bewirken. Und in der Tat ist es die größte Herausforderung für außerschulische Bildungsanbieter, langfristig Bildungsangebote aufrechterhalten zu können. Sie benötigen ein Netzwerk mit Partnern und finanziellen Ressourcen.

Regionales Lernen 21+ ist ein Konzept für außerschulisches, handlungsorientiertes Lernen. Es wurde im Landkreis Vechta entwickelt und international erprobt und evaluiert (Schockemöhle, 2009). Es folgt dem Leitbild der BNE und

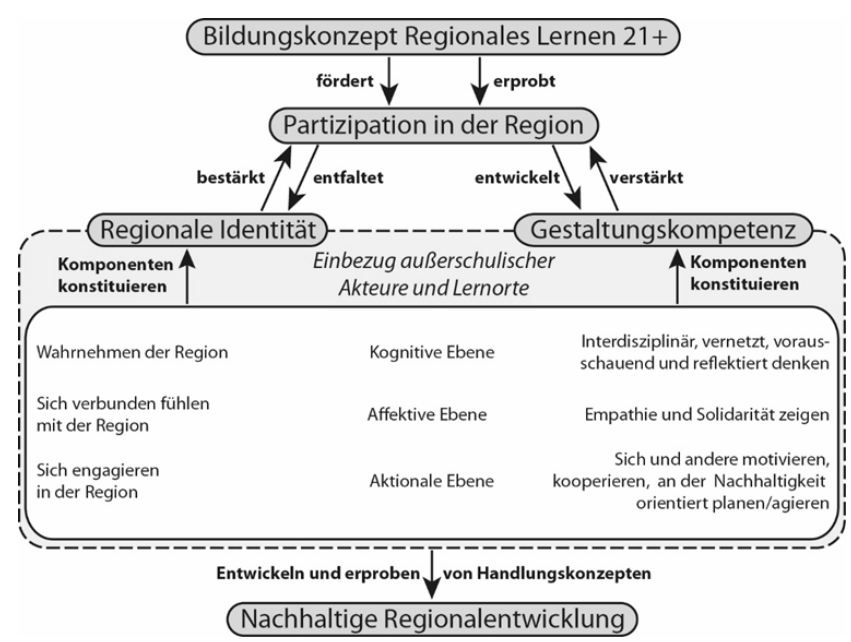

Abb. 1: Zentrale Kennzeichen des Bildungskonzepts Regionales Lernen 21+, Quelle: verändert nach Schockemöhle, 2009, S. 96

bindet außerschulische Menschen und Orte des Nahraums in Bildungsangebote ein. Die Region ist der Lernraum, in dem für ausgewählte Themen Schülerinnen und Schüler eine lebensweltbezogene Auseinandersetzung erfahren und erleben. So entstand seit dem Jahr 2005 ein Bildungsnetzwerk zur Land- und Ernährungswirtschaft im Landkreis Vechta. Etwa 40 Lernorte des Kreises, in dem Land- und Ernährungswirtschaft raum- und wirtschaftsprägende Elemente sind, sind involviert. 36 Schulen sind als Partnerschulen dabei. Im Kern finden außerschulische Lernangebote für Kinder und Jugendliche jeden Alters und jeder Schulform statt. So lernen sie die Land- und Ernährungswirtschaft in der Realbegegnung kennen und bekommen Einsicht in Themen, Herausforderungen und Partizipationsmöglichkeiten. Schockemöhle und Duda konnten nachweisen, dass es gelingt, Gestaltungskompetenz, die regionale Identität und das vernetzte Denken durch die Lernangebote zu fördern (Duda, 2014; Schockemöhle, 2009).

In der Zwischenzeit haben sich weitere Regionen auf den Weg gemacht, Bildungsnetzwerke zu entwickeln, die durch regionsbezogene Bildungsarbeit einen Beitrag zur nachhaltigen Regionalentwicklung leisten möchten. Die Region Weser-Ems thematisiert das Leitbild Bioökonomie im Rahmen der (außer-)schulischen Studien- und Berufsorientierung. Die Region Niedersächsische Nordseeküste stellt die Fischwirtschaft in den Mittelpunkt ihres Bildungsnetzwerks. Kinder und Jugendliche erfahren so die Möglichkeit, direkt an den Entwicklungen in der Region teilhaben zu können, bekommen Einblicke und Kontakte, um kurz-, mittel- und langfristig zu partizipieren.

In Niedersachsen sind über sechzig Bildungseinrichtungen als „außerschulische Lernstandorte BNE“ vom Niedersächsischen Kultusministerium anerkannt worden (Niedersächsisches Kultusministerium, 2019). Überwiegend handelt es sich um die vom Land Niedersachsen eingerichteten Regionalen Umweltbildungszentren (RUZ), aber auch weitere Institutionen der Umweltbildung. Es ist ein fortlaufender Prozess angestoßen worden, der die Bewerbung weiterer Institutionen zulässt. Der Auswahl zugrunde liegt ein umfangreicher Kriterienkatalog, der den Bezug zum Leitbild BNE widerspiegelt. 
„Außerschulische Lernorte BNE“ in Österreich Best-Practice-Beispiele für Österreich finden sich zum Beispiel im Bereich Gartenbasierten Lernens. Gartenbasiertes Lernen und Arbeiten ist eine interdisziplinäre und altersstufenunabhängige Methode, die den Garten als Ressource und Lehrmittel für inklusive Bildungsprozesse nutzt. Dabei umfasst das Gartenbasierte Lernen verschiedene Programme, Aktivitäten und Projekte, bei denen der Garten und die damit verbundene gärtnerische Praxis die Grundlage für ein neugieriges, eigenverantwortliches und handlungsorientiertes Lernen darstellt (Desmond et al., 2002, S. 7).

Mittels fachkundiger Anleitung und Aufforderung zur Reflexion wird im Gartenbasierten Lernen sinnstiftendes Wissen und Erfahrung so miteinander verknüpft, dass die Lernenden ein Umweltbewusstsein entwickeln, welches auf Nachhaltigkeit und ressourcenschonendes Verhalten abzielt (Haubenhofer \& Wolf, 2015). Wie Haubenhofer, Demattio \& Geber (2012) in ihrer Recherche und Analyse fachbezogener Artikel zur Wirkung und zum Nutzen von Green Care aufzeigen konnten, hat das Gartenbasierte Lernen positive Effekte auf die Einstellung zur Umwelt, auf das Ernährungsverhalten, die soziale Zusammenarbeit und die interpersonelle Kompetenz von Menschen, sowie auf deren Aneignung von Fachwissen. Im Folgenden werden zwei solche österreichischen Gartenpädagogikprojekte vorgestellt.

Das Projekt „Education in Gardens" umfasst renommierte Projektpartner aus Österreich und der Tschechischen Republik, die gemeinsam neue und grenzüberschreitende Bildungsprogramme für Pädagoginnen, Pädagogen, Studierende, Schülerinnen und Schüler für das Lernen im Garten entwickeln und anschließend grenzüberschreitend und nachhaltig in der Bildungslandschaft verankern. Hierzu werden im Rahmen des Projektes Weiterbildungen und Workshops für Pädagoginnen, Pädagogen und Pädagogikstudierende angeboten (Wappel \& Kropf, 2018).

Weil das Projekt nicht nur die manuelle Geschicklichkeit, die Sinneswahrnehmung, die persönliche Entwicklung sowie das Interesse an Umweltthemen und an naturwissenschaftlichen Fächern bei den Lernenden fördern will, sondern sich auch explizit am Leitbild der nachhaltigen Entwicklung orientiert, erhielt es vom österreichischen Bundesministerium für Nachhaltigkeit und Tourismus die Auszeichnung „Bildung für nachhaltige Entwicklung - BEST OF AUSTRIA“. Denn „Education in Gardens“ legt großen Wert auf biologische Vielfalt, die Förderung heimischer und ökologisch wertvoller Pflanzen sowie die Vermittlung von Wissen und Fertigkeiten zur ökologischen und naturnahen Gestaltung und Pflege von Gärten. Dabei deckt sich das Leitbild des Projekts „,gesund halten was uns gesund erhält" mit dem Kerngedanken der BNE, wonach die Lebensgrundlage Boden auch für zukünftige Generationen in einer guten Qualität zu erhalten ist (Wappel \& Kropf, 2018).

Die im Projekt „Education in Gardens“ entwickelten Lernsettings setzen auf Mehrperspektivität und Transdisziplinarität. Indem naturnahe Gärten mit ihren Natur- und Stoffkreisläufen und ihrer biologischen Vielfalt als Lernraum zur Vermittlung von theoretischen Kenntnissen und praktischen Fertigkeiten genutzt werden, lässt sich gärtnerisches und pädagogisches wie auch fächerspezifisches und fächerverbindendes
Arbeiten in Lernsettings zu naturwissenschaftlichen und umweltrelevanten Themen, verbinden. Dabei stellen Flow-learning $^{1}$ (Cornell et al., 2017, S. 33) und das projektbezogene Lernen wichtige, die Mehrperspektivität fördernde Methoden in der Gartenpädagogik dar (Wappel \& Kropf, 2018).

Die City Farm Augarten ist ein gemeinnütziger Verein zur Förderung von Urban Gardening, Gartenpädagogik und ökologischer Bildung. Geplant und umgesetzt nach dem Vorbild großer, internationaler Institutionen wie dem Brooklyn Botanic Garden in New York, nahm die City Farm Augarten im Jahr 2012 ihre Arbeit auf. Mittlerweile kann sie jährlich 4.500 Kinder und 1.000 Erwachsene mit ihren gartengestützten Bildungsangeboten erreichen (City Farm Schönbrunn, 2017). In urbaner Umgebung, mitten im zweiten Wiener Gemeindebezirk gelegen, bietet der im Jahr 2018 neu bezogene Standort Augarten Kindergärten und Schulen die Möglichkeit, ganzjährig das saisonal angepasste gartenpädagogische Programm zu naturwissenschaftlichen und umweltrelevanten Themen aufzusuchen. Der Besuch der City Farm soll dabei nicht nur gärtnerische Fähigkeiten unterstützen, sondern auch Bewegung in und mit der Natur, gesunde Ernährung, soziale Kompetenz sowie Achtsamkeit gegenüber Lebensmitteln und der Natur fördern (City Farm Schönbrunn, 2017). Hierfür bietet die City Farm gartenpädagogische Programme zu monatlich wechselnden Themenschwerpunkten an. Die inhaltliche Aufbereitung des Themas orientiert sich dabei einerseits an der Programmart, andererseits am Alter der Workshopbesucherinnen und -besucher.

\section{Inklusion beim außerschulischen Lernen}

Seit der Verpflichtung Deutschlands zur Umsetzung der UN-Konvention im Hinblick auf ein inklusives Bildungssystem im Jahr 2009 ist vieles in Bewegung: Dem Leitbild der Inklusion folgend, sind alle Bildungsinstitutionen gefordert, nach immer besseren Wegen zu suchen, Lernprozesse in heterogenen Lerngruppen zu gestalten. Ziel ist es, die Leistungen und Partizipationsmöglichkeiten aller Schülerinnen und Schüler möglichst effektiv zu unterstützen und spezifische Entwicklungs- und Lernmöglichkeiten für Lernende in heterogenen Lerngruppen zu schaffen.

Die Forschungswerkstatt Inklusion (Universität Vechta, Projekt BRIDGES) hat im interdisziplinären Diskurs Merkmale für eine gute inklusive Schule und einen guten inklusiven Unterricht entwickelt. Zugrunde liegt ein Verständnis von Inklusion, indem die Vielfalt und das Miteinander von verschiedenen Menschen in einem gemeinsamen Kontext als bereichernd angesehen werden: „Inklusion bezeichnet Basiswerte der Gesellschaft: Partizipation ist ein Menschenrecht. Alle Menschen werden in ihrer Vielfalt und Individualität wahrgenommen, angenommen und wertgeschätzt. Die Vielfalt wird als Ressource wahrgenommen. Bezogen auf Bildung in der Schule und dort speziell im Unterricht bedeutet für uns Inklusion, auf Bedürfnisse von Gesellschaft und Individuen einzugehen sowie individuelle Lernvoraussetzungen zu erkennen, zu berücksichtigen und dementsprechend zu fördern. Individualisierung und Gemeinschaft sind dabei gleichermaßen wichtig" (Baumert, Vierbuchen \& Team BRIDGES, 2018, S. 526). Für die inklusive Schule wurden die Merkmale Haltung, Gemein- 
schaft und Kooperation, Ressourcen, Barrierefreiheit und Kommunikation herausgestellt. Für außerschulische Bildungsinstitutionen sind diese direkt übertragbar. Bezogen auf die pädagogische Haltung braucht es Wertschätzung gegenüber allen Lernenden und die Reflexion und Vermeidung von Stigmatisierungen. Gemeinschaft und Kooperation stehen für ein Bildungskonzept, welches offene, kooperative und projektorientierte Lernformen beinhaltet und die Partizipation und Gemeinschaft fördert. Die Ausstattung mit für inklusive Bildungsarbeit erforderlichen personellen, finanziellen und materiellen Ressourcen ist ebenfalls grundlegend. Barrieren zeigen sich in baulicher, kommunikativer und sozialer Form. So können diskriminierende Haltungen und schwierige Sprache zum Ausschluss von Schülerinnen und Schülern führen. Das Kriterium Kommunikation adressiert schließlich das Sprachverhalten der Lehrenden als grundlegende pädagogische Qualität, welches die vorhandene Empathie und Wertschätzung transportieret. Für den guten inklusiven Unterricht wurden weitere vierzehn Qualitätskriterien identifiziert: Klassenführung, effektive Lernzeit, lernförderliches und vertrauensvolles Klima, vielfältige Motivierung, kognitive Aktivierung, Klarheit und Struktur, Schüler- und Kompetenzorientierung, Sprache und Sprachsensibilität, individuelles Fördern, individuelles Feedback, interne Kooperationen, vorbereitete Lernumgebung, Angebotsvielfalt, individuelle und transparente Leistungserwartung (Baumert et al., 2018, S. 528f.). Sie sind ebenfalls auf das außerschulische Lernen übertragbar. Aus dem Blickwinkel der Zusammenarbeit zwischen außerschulischen Bildungsanbietern und Schulen zeigt sich jedoch, dass die kursiv gesetzten Kriterien besonders bedeutsam sind. Außerschulische Lernphasen unterstützen Schulen darin, das Interesse von Schüle- rinnen und Schülern für bestimmte Themen zu wecken, die Bedeutsamkeit aufzuzeigen, den Lebensweltbezug herzustellen und ggfs. eine Identitätsbildung zu fördern (vielfältige Motivierung). Außerschulische Lernorte verfügen über eine anregende, strukturierte Lernumgebung, die individuelle und kooperative Lernprozesse fördert. Barrierefreiheit, Mitgestaltungsmöglichkeiten und ergometrische Bedingungen (Mobiliar, Beleuchtung, Belüftung und Akustik) sind weitere Qualitätsfaktoren beim Kriterium „vorbereitete Lernumgebung“. Bei der „Angebotsvielfalt“ können außerschulische Bildungsanbieter ausgefeilte Lernangebote mit einem hohen Grad an Methodenvielfalt, mit kooperativem und individuellem Lernen, mit offenem und strukturiertem Lernen, mit der Ansprache aller Sinne und mit verschiedenen Erfahrungs- und Erlebnismomenten bieten.

Der Bereich der außerschulischen Bildung ist gegenüber dem öffentlichen schulischen Bildungssektor sehr viel stärker von schlechten Rahmenbedingungen betroffen. Es werden kaum flankierende Maßnahmen entwickelt und durchgeführt, die hier unterstützend wirken können. Außerschulische Bildungsangebote können jedoch dazu beitragen, die Rahmenbedingungen für Inklusion an Schulen zu verbessern, indem sie Lernumgebungen schaffen, die das spezifische Potenzial für inklusive Lerngruppen voll ausschöpfen. Denn durch die vielfältigen Möglichkeiten der originalen Begegnung mit Lerngegenständen und einen handlungsorientierten Ansatz wird ein Lernen mit allen Sinnen möglich, welches Lernwege, Lernmotive und Interesse schafft, das am Lernort Schule in vergleichbarer Weise nicht möglich ist. In Tabelle 1 finden sich grundsätzliche Überlegungen, wie außerschulische Bildungsanbieter sich auf eine inklusive Bildungsarbeit einstellen können.

\begin{tabular}{|l|l|l|}
\hline \multicolumn{1}{|c|}{ Merkmal } & \multicolumn{1}{|c|}{ Ausprägung } & \multicolumn{1}{|c|}{ Gestaltungsbeispiel } \\
\hline Räumlichkeiten und Gelände & $\begin{array}{l}\text { Zugänglichkeit und Teilhabemöglichkei- } \\
\text { ten für alle }\end{array}$ & $\begin{array}{l}\text { Zugänglichkeit/Barrierefreiheit von Na- } \\
\text { turarealen an Umweltbildungszentren } \\
\text { für Menschen mit körperlichen Beein- } \\
\text { trächtigungen ist vorhanden. }\end{array}$ \\
\hline Bildungskonzept & Inklusion als Leitbild ist implementiert & $\begin{array}{l}\text { Überzeugung, dass inklusives Lernen } \\
\text { sich eignet, die Gemeinschaft zu fördern, } \\
\text { Werte zu entwickeln und Leistungen zu } \\
\text { steigern, ist im Leitbild vorhanden. }\end{array}$ \\
\hline Bildungsangebote & $\begin{array}{l}\text { Angebote, die heterogene Lerngruppen } \\
\text { in Inhalt und Durchführung adressieren }\end{array}$ & $\begin{array}{l}\text { Bedarfe von Teilnehmenden mit Beein- } \\
\text { trächtigungen werden aufgegriffen. }\end{array}$ \\
\hline Personal & $\begin{array}{l}\text { Die Anbieter sind geschult für inklusive } \\
\text { Lerngruppen. Sie können individuell auf } \\
\text { (pädagogische Mitarbeiterinnen und } \\
\text { Mitarbeiter, Lehrpersonen) }\end{array}$ & $\begin{array}{l}\text { Das Personal ist in Qualität und Quan- } \\
\text { tität angemessen vorhanden, um hetero- } \\
\text { gene Gruppen betreuen zu können (z.B. } \\
\text { multiprofessionelle Teams, kleine Lern- } \\
\text { gruppen). Das Personal bildet sich re- } \\
\text { gelmäßig fort. Es steht ausreichend Zeit } \\
\text { zur Verfügung, um auf die spezifischen } \\
\text { Anforderungen inklusiver Lerngruppen } \\
\text { reagieren zu können. }\end{array}$ \\
\hline Finanzierung
\end{tabular}


Nur sehr wenige außerschulische Bildungsinstitutionen verfügen über Jahrzehnte lange Erfahrung mit der Bildungsarbeit mit Menschen mit Behinderung. Diese Leuchttürme bieten heute jedoch die Möglichkeit, praxisnahe Erfahrungen aufzugreifen und von ihnen zu lernen. Ein Beispiel ist das Zentrum für Erlebnispädagogik und Umweltbildung e.V. (ZERUM) in Ueckermünde. Das ZERUM ist ein Schullandheim und eine Jugendbildungseinrichtung direkt am Stettiner Haff in Mecklenburg-Vorpommern gelegen. Die Schwerpunkte der Einrichtung liegen im Bereich der Abenteuer- und Erlebnispädagogik, der handlungsorientierten Umweltbildung und der Integration von jungen Menschen mit Beeinträchtigungen. Beeindruckend zeigt sie, wie Teilhabe gelingen kann: Alle Menschen können - auch mit Rollstuhl - ans Meer, auf das Schiff und in den Kletterwald. Alle Barrieren wurden überwunden, um die grundlegenden Erlebnisse und Erfahrungen allen Teilnehmenden zu ermöglichen. Das ZERUM zeigt, dass die Haltung, der Wille zur Inklusion und die Wahrnehmung von Barrieren jeglicher Art wesentliche Fundamente zur inklusiven Bildungsarbeit sind. Die meisten außerschulischen Bildungsanbieter befassen sich jedoch im Vergleich zu den Allgemeinbildenden Schulen erst seit vergleichsweise kurzer Zeit mit dem Ziel und der gesellschaftlichen Verpflichtung zur Inklusion. Daher ist zu beobachten, dass in der Praxis zwar häufig räumliche Barrieren abgebaut sind: Eine Rampe schafft für Rollstuhlfahrer den Zugang anstelle von Treppenstufen. Tafeln mit Blindenschrift sind zu finden. Parkplätze wurden geschaffen. Dieses trifft allerdings für den Zugang ins Freigelände beim außerschulischen Lernen häufig nicht zu. Der Kletterpark, die Waldexkursion oder der Besuch der Streuobstwiese sind häufig nicht für alle erreichbar. Da der Handlungsdruck an Schulen sehr viel höher ist und die Unterstützung durch Dritte ebenfalls, sind die außerschulischen Bildungsanbieter grundsätzlich schlechter auf die Anforderungen der Inklusion eingestellt. Grundsätzlich ist eine große Vielfalt zu beobachten: von bereits erfahrenen Einrichtungen wie dem ZERUM bis hin zu Anbietern, die bisher gar keine Veränderungen vorgenommen haben.

Ein Promotionsvorhaben der Forschungswerkstatt Inklusion widmet sich der Erforschung des inklusiven Geogra- phieunterrichts mit außerschulischen Phasen. Die Arbeit von Tiller hat den Titel „Regionales außerschulisches Lernen im inklusiven Geographieunterricht - Das Potenzial des Bildungskonzeptes des Regionalen Lernens 21+ für die Förderung der Berufsorientierung im Geographieunterricht unter besonderer Berücksichtigung von inklusiven Lerngruppen“. Wie im Gesamtprojekt der Forschungswerkstatt Inklusion geht es um die Identifizierung und Beschreibung von Gelingensbedingungen und Qualitätskriterien für inklusives Lernen - hier jedoch speziell für den Bereich des außerschulischen Lernens. An einem selbst entwickelten Beispielmodul zur Berufsorientierung im Geographieunterricht durch Betriebserkundungen erforscht Tiller das Potenzial außerschulischen, regionalen Lernens für die Inklusion. Mit einem Mixed-Methods-Design (Döring \& Bortz, 2016) werden zudem die Lernprozesse analysiert (Tiller, im Druck). Außerschulisches Lernen und die Kooperation mit außerschulischen Bildungsanbietern können durch die Anschaulichkeit der Realbegegnung das Lernen mit allen Sinnen und den hohen Grad der Handlungsorientierung sowie einem hohen Niveau der didaktisch-methodischen Gestaltung ein unterstützendes Element inklusiver schulischer Bildung sein. Grundsätzlich sind an außerschulisches Lernen die gleichen Qualitätsanforderungen wie an schulisches Lernen zu stellen. Aufgrund der unterschiedlichen Potenziale dieser Lernformen sind jedoch besonders die oben ausgeführten Qualitätskriterien von Bedeutung.

\section{Kooperationen zwischen Lehrkräften und außerschulischen Lernorten}

Weil Inklusion in Erziehung und Bildung nicht nur den Abbau von Barrieren für die Teilhabe aller Schülerinnen und Schüler am schulischen und außerschulischen Lernen meint, sondern auch die gleiche Akzeptanz und Wertschätzung für alle Lernenden, das Sichtbarmachen der sich aus der Inklusion für alle Beteiligten ergebenden Chancen sowie den Auf- und Ausbau langfristiger Beziehungen zwischen Schule und ihrem sozialräumlichen Umfeld (Boban \& Hinz, 2003, S. 10), reicht es nicht aus, nur die Rahmenbedingungen, Strukturen und Ab-

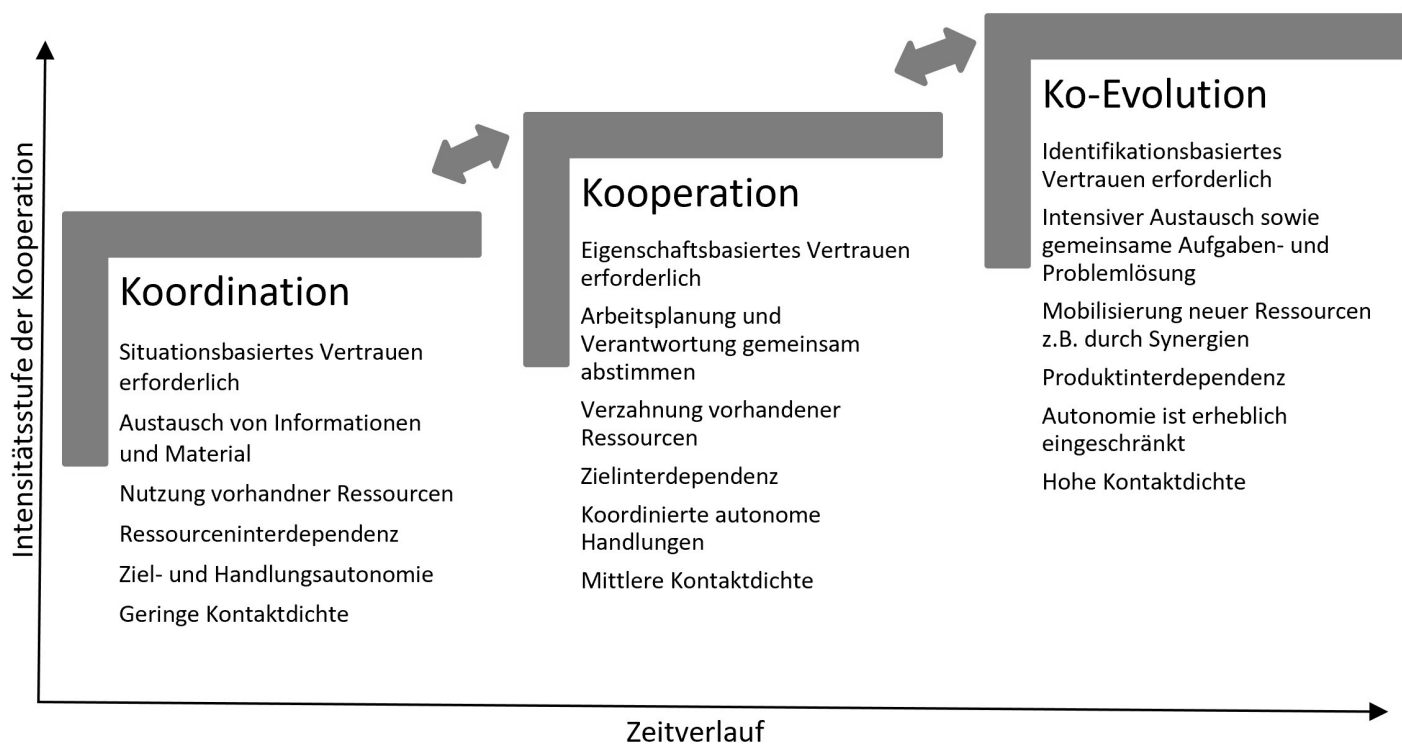

Zeitverlauf 
läufe in Unterricht und Schule anzupassen (Pauser, 2009, S. 153f.). Vielmehr braucht es Absprachen und Vereinbarungen zwischen der Schule und den außerschulischen Lernorten, so dass auch an diesen Lernorten ein inklusives, pädagogisches Handeln sowie eine Lernkultur ermöglicht wird, in der inklusive Haltungen, Werte, Strukturen und Praktiken des Unterrichts und der Ressourcenmobilisierung verankert sind (Boban \& Hinz, 2003, S. 14). Damit solche Absprachen möglich werden und anschließend langfristig Wirkung zeigen, muss die kontinuierliche Zusammenarbeit zwischen Schule und außerschulischem Lernort sichergestellt werden. Die Kooperierenden können dabei selbst entscheiden, welche Kooperationsintensität (Abb. 2) sie als geeignet erachten, um ihre Kooperationsziele zu erreichen.

Interprofessionelle Kooperationen, in denen die Lehrkraft, der (eventuell eingesetzte) Integrationshelfer oder die Integrationshelferin, die Pädagogin oder der Pädagoge des außerschulischen Lernorts jeweils ihre unterschiedlichen persönlichen Kompetenzen, Fähigkeiten und Ressourcen einbringen und miteinander verknüpfen, eröffnen den Kooperierenden den Zugang zu neuen Informationen (Helmcke, 2008, S. 50). So können Lehrkräfte zum Beispiel Informationen über den außerschulischen Lernort von den außerschulischen Pädagoginnen und Pädagogen erhalten. Umgekehrt erfahren diese etwas über die Bedürfnisse und die Heterogenität der Gruppe und welche spezielle Unterstützung einzelne Schülerinnen und Schüler benötigen.

Des Weiteren sind komplexe Problemstellungen, die sich nur zusammen mit einem Kooperationspartner lösen lassen, eine Spezialität von Kooperationen (Helmcke, 2008, S. 54). Die Planung und Durchführung von außerschulischen Lehr-Lern-Einheiten, die heterogene Lerngruppen in Inhalt und Durchführung adressieren, könnte so eine komplexe Problemstellung sein, die sich nur in der engen Zusammenarbeit und dem Austausch zwischen allen Kooperierenden lösen lässt.

Weiterer Kooperationsnutzen erwächst aus der Möglichkeit, in der Kooperation komplementäre Ressourcen zu bündeln (Helmcke, 2008, S. 56). So bringen die Kooperierenden ihre unterschiedlichen persönlichen Kompetenzen (ihr Fachwissen, ihre Erfahrungen, ihre Kontakte etc.) in die Kooperation ein. Zudem bieten außerschulische Lernorte vielfältige Gelegenheiten, damit die Schülerinnen und Schüler ihre menschliche Mitwelt, sachliche Umwelt und deren gegenseitige Bezüge erleben, entdecken, beobachten, erfahren und erforschen können (Flath, 2011, S. 154f.).

Eine weitere Stärke von Kooperationen zeigt sich im Innovationslernen, welches immer dann stattfindet, wenn die Erfahrungen und Kompetenzen der Kooperierenden zu neuen Ideen und Einsichten verknüpft werden (Helmcke, 2008, S. 56f.). Dieses kann in der Kooperation zwischen Lehrkraft, der Integrationshelferin bzw. dem Integrationshelfer und dem pädagogischen Personal des außerschulischen Lernorts zum Beispiel bei der Planung einer außerschulischen Lehr-LernEinheit stattfinden, wenn alle Beteiligten ihre Ideen und ihr Wissen austauschen und so zur Konzeption einer inklusiven Lehr-Lern-Einheit eng miteinander verknüpfen.

Damit diese interprofessionellen Kooperationen zwischen der Lehrkraft, dem (eventuell eingesetzten) Integrationshelfer oder der Integrationshelferin, der Pädagogin oder dem
Pädagogen des außerschulischen Lernorts gelingt, sind die folgenden Bedingungen zu berücksichtigen:

- FürdieEntwicklungvon Vertrauen und einer gemeinsamen Kooperationskultur ist es erforderlich, dass sich die Kooperierenden über ihre Normen, Werte, Regelungen, Bedürfnisse, Motive, Ziele und Arbeitsformen aber auch über die eingebrachten unterschiedlichen Zeit- und Finanzressourcen austauschen.

- Für die Entwicklung der Bereitschaft und Fähigkeit zur Zusammenarbeit benötigen die Kooperationspartner Kommunikationskompetenz, Offenheit, Wertschätzung, Reziprozität, Gleichberechtigung, Irritationsfreundlichkeit, Kreativität, Toleranz- und Konfliktfähigkeit (inkl. Strategien zum Konfliktmanagement und die Bereitschaft zum Konsens) sowie empathisches Vermögen.

- Eine Gelingensbedingung ist die Bereitschaft bei klar abgegrenzten fachlichen Identitäten voneinander zu lernen. Dies betrifft sowohl den Erwerb von Grundkenntnissen und Grundqualifikationen in den Bereichen, die für die Gestaltung von Lehr-Lern-Einheiten auf außerschulischen Lernorten von Nöten sind, als auch Kenntnisse über die Fachkompetenzen der anderen Kooperationspartner.

- Eine positive Einstellung gegenüber der Kooperation stellt eine weitere Gelingensbedingung für eine erfolgreiche Zusammenarbeit dar. Diese kann durch eine realistische Arbeitsplanung und das Erleben, dass den Kooperationspartnern die Zusammenarbeit nützt, gefördert werden.

- Die Verankerung der Kooperationsaktivitäten in den Strukturen der jeweiligen Herkunftsorganisation ist ebenfalls erforderlich, damit die Kooperationen langfristig gesichert sind.

Sind solche Kooperationen erfolgreich, so ist deren „Lohn“ nicht nur die Ermöglichung inklusiver Lehr-Lern-Arrangements an außerschulischen Lernorten, sondern darüber hinaus die Förderung der Kontinuität der Lernortbesuche, die Ausrichtung der außerschulischen Lehr-Lern-Arrangements an den didaktischen Prinzipien der Handlungs- und Problemorientierung sowie deren Vor- und Nachbereitung im Unterricht, was wiederum einen signifikanten Einfluss auf die Förderung von regionaler Identität und Gestaltungskompetenz (BNE) hat (Diersen \& Flath, 2016b, S. 216; Schockemöhle, 2009, S. 288).

\section{Zusammenführung der Ergebnisse und Ausblick}

Betrachtet man die bisherigen Analyse- und Bewertungsergebnisse zum außerschulischen Lernen, der BNE und der Inklusion unter Berücksichtigung der eingangs gestellten Fragestellung - inwiefern außerschulisches Lernen einen Beitrag für eine Bildung für nachhaltige Entwicklung (BNE) und eine inklusive Gesellschaft leistet - so ergibt sich folgendes Fazit. BNE und Inklusion stehen für Entwicklungen im Bildungssystem, die bisher weitgehend parallel und unabhängig voneinander verlaufen. Dabei verfolgen sie in Teilen gleiche Ziele. Sie möchten Partizipation und Teilhabe fördern, um eine gesellschaftliche Entwicklung bzw. Transformation zu erreichen. 
Die gesetzten Oberziele unterscheiden sich. So adressiert die BNE gesamtgesellschaftliche Entwicklungen mit dem Hauptziel ökologische, aber auch soziale Herausforderungen zu lösen. Die Inklusion verfolgt das Ziel der gleichberechtigten Teilhabe aller Menschen.

Beide Leitbilder sind als Querschnittsthemen moderner Pädagogik angelegt. Zurzeit werden sie jedoch häufig additiv in Form von Projekten und Maßnahmen realisiert. Beim außerschulischen Lernen ist BNE onmipräsent, während die Inklusion bisher vergleichsweise wenig nach außen wahrnehmbare Berücksichtigung findet. Letzteres ist durch die häufig nur punktuelle Zusammenarbeit mit Schulen zu erklären, wodurch ein gezieltes pädagogisches Arbeiten mit inklusiven Schülerinnen und Schülern kaum möglich ist. Vor allem aber ist es auch eine Frage der in der Regel geringen finanziellen und personellen Ressourcen der außerschulischen Bildungsanbieter.

Es wurde gezeigt, dass mit einer in den Unterricht integrierten und diesen ergänzenden Nutzung außerschulischer Erfahrungs-, Lern- und Handlungsräume, dem Problem der mangelnden Erfahrbarkeit oder des fehlenden Sinnbezugs von Lerninhalten begegnet werden kann. Zugleich wirkt außerschulisches Lernen der zunehmenden Naturentfremdung von Kindern und Jugendlichen entgegen und fördert darüber hinaus deren Selbstständigkeit, Neugierde, Kreativität, Entdeckerdrang wie auch deren Fähigkeiten zur Selbstorganisation und zum vorausschauenden, reflektierten, vernetzten und interdisziplinären Denken. Zudem leistet außerschulisches Lernen einen wichtigen Beitrag zur BNE und zur Berufsorientierung. Hierbei ist wiederum zu bedenken, dass gerade die Berufstätigkeit einen hohen BNE-Bezug aufweist. Denn Menschen handeln nicht nur privat im Sinne einer nachhaltigen Entwicklung, sondern auch in hohem Maße beruflich. Nicht zuletzt besitzt Lernen an außerschulischen Lernorten ein noch nachzuweisendes hohes Potenzial für Inklusion als Ergänzung zum schulischen Lernen. Soll all dieses Potenzial außerschulischen Lernens wirksam werden, so braucht es:

- eine Kontinuität in der Zusammenarbeit zwischen Schule und außerschulischen Lernorten,

- eine Unterstützung für die Lehrperson bei der Planung und Organisation des Lernortbesuchs,

- eine regelmäßig wiederkehrende Nutzung außerschulischer Lernorte,

- eine an den beschriebenen didaktischen Prinzipien ausgerichtete Durchführung außerschulischer Lehr-Lern-Arrangements und

- Rahmenbedingungen, die gewährleisten, dass alle Lernenden in ihrer Vielfalt und Individualität wahrgenommen, angenommen und wertgeschätzt werden und auf deren unterschiedliche Bedürfnisse eingegangen wird sowie deren individuelle Lernvoraussetzungen Berücksichtigung finden.

\section{Anmerkungen}

1 Das Flow-Learning-Konzept des US-amerikanischen Naturpädagogen Joseph Cornell beschreibt, wie Menschen mittels vier, aufeinander aufbauender und fließend ineinander übergehender Stufen (Begeisterung wecken, konzentriert wahrnehmen, unmittelbare Erfahrungen machen und andere an diesen Erfahrungen teilhaben lassen) eine respektvolle und wertschätzende Beziehung zu ihrer natürlichen Umwelt aufzubauen lernen (Cornell et al., 2017, S. 33).

\section{Literatur}

Baar, R. \& Schönknecht, S. (2018). Außerschulische Lernorte: didaktische und methodische Grundlagen. Weinheim: Beltz Pädagogik.

Baumert, B., Vierbuchen, M.-C. \& Team BRIDGES (2018). Eine Schule für alle - Wie geht das? Qualitätsmerkmale und Gelingensbedingungen für eine inklusive Schule und inklusiven Unterricht. Zeitschrift für Heilpädagogik, 69(11), 526-541. Zugriff am 02.09.2019 https://www.uni-vechta.de/fileadmin/user_upload/Zen trum_fuer_Lehrerbildung/ProjektBridges/02_Dateien/zfh_11.2018_baumertvierbuchen.pdf

Boban, I. \& Hinz, A. (2003). Index für Inklusion. Lernen und Teilhabe in der Schule der Vielfalt entwickeln. Halle an der Saale: Martin-Luther-Universität Halle-Wittenberg. Zugriff am 10.11.2019 https://www.eenet.org.uk/resources/docs/Index\%20 German.pdf

Bund \& Misereor (Hrsg.) (1996). Zukunftsfähiges Deutschland. Ein Beitrag zu einer global nachhaltigen Entwicklung. Basel: Birkhäuser. https://doi.org/10.1007/978-30348-6070-3

Christen, O. (1999). Nachhaltige Landwirtschaft. Bonn: Gesellschaft zur Förderung des integrierten Landbaus $\mathrm{mbH}$.

City Farm Schönbrunn (2017). Projektplan. Wien: City Farm Augarten.

Cornell, J., Louv, R. \& Song, T. (2017): Cornells Naturerfahrungsspiele für Kinder und Jugendliche. Die besten Klassiker und neue Spiele. Sonderedition. Mülheim an der Ruhr: Verlag an der Ruhr.

Desmond, D., Grieshop, J. \& Subramaniam, A. (2002). Revisiting garden based learning in basic education. Philosophical roots, historical foundations, best practices and products, impacts, outcomes and future directions. Paris: Food and Agriculture Organization/United Nations International Institute for Educational Planning.

Deutsche Gesellschaft für Geographie (Hrsg.) (2017). Bildungsstandards im Fach Geographie für den Mittleren Schulabschluss mit Aufgabenbeispielen (9. Auflage). Bonn: Selbstverlag Deutsche Gesellschaft für Geographie. Zugriff am 02.09.2019 http://geogra phiedidaktik.org/wp-content/uploads/2017/10/Bildungsstandards_ Geographie_9.Aufl_._2017.pdf

Deutsche UNESCO-Kommission e.V. (o.J.). UNESCO-Weltaktionsprogramm: Bildung für nachhaltige Entwicklung. Die UN-Dekade BNE. Zugriff am 05.11.2019 https://www.bne-portal.de/de/bundesweit/un-dekade-bne-2005-2014

Diersen, G. \& Flath, M. (2016a). Regionales Lernen 21+- Konzept, Wirkung und Stellenwert im fächerübergreifenden außerschulischen Lernen. transfer. Forschung $\leftrightarrow$ Schule. Visible Didactics - Fachdidaktische Forschung trifft Praxis (2),179-188.

Diersen, G. \& Flath, M. (2016b). Regionales Lernen 21+. Konzept, Wirkung und Anwendung. In C. Berndt,C. Kalisch \& A. Krüger (Hrsg.), Räume bilden - pädagogische Perspektiven auf den Raum (S. 214-224). Bad Heilbrunn: Julius Klinkhardt.

Diersen, G. \& Flath, M. (Hrsg.) (2018). Die Streuobstwiese - ein Hit in der Kulturlandschaft. Handlungsorientierte Lehr-Lernmaterialien für die Grundschule und die Klassenstufen 5-10 unter Berücksichtigung der Herausforderungen inklusiven, außerschulischen Unterrichts. Zugriff am 21.10.2019 https://www.uni-vechta.de/fileadmin/user_upload/ISPA/Publikationen/Weisse_Reihe/Streuobstwiese_ Stand_17.12.2018.pdf

Dillon, J., Rickinson, M., Teamey, K., Morris, M., Choi, M.Y., Sanders, D. \& Benefield, P. (2006). The value of outdoor learning: evidence from research in the UK and elsewhere. School Science Review, 87(320), 107-111.

Döring, N. \& Bortz, J. (2016). Forschungsmethoden und Evaluation in den Sozialund Humanwissenschaften. Berlin \& Heidelberg: Springer. https://doi.org/10.1007/ 978-3-642-41089-5

Duda, C. (2014). Ganztagsbildung und das Konzept des Regionalen Lernens 21+. Geographiedidaktische Forschungen Band 52. Weingarten: Selbstverlag Hochschulverband für Geographiedidaktik.

Dziewas, A. (2008). Chemieunterricht an außerschulischen Lernorten. Duisburg \& Essen: Fakultät für Chemie der Universität Duisburg-Essen.

Enquete-Kommission „Schutz des Menschen und der Umwelt“ (1998). Konzept Nachhaltigkeit. Vom Leitbild zur Umsetzung. Abschlussbericht der Enquete- Kommission „Schutz des Menschen und der Umwelt" des 13. Deutschen Bundestages. Bonn: Deutscher Bundestag.

Flath, M. \& Rudyk, E. (2017). Geo-Aktiv. Wir erkunden ein Unternehmen in unserer Region. In Flath, M. \& Rudyk, E. (Hrsg.), Unsere Erde (Differenzierende Ausgabe). Niedersachsen 9|10 (S. 130-131). Berlin: Cornelsen. 
Goller, H. (2002). Kontextabhängiger Erwerb von Arten- und Formenkenntnissen im Biologieunterricht des Gymnasiums. Regensburg: Hochschulschrift der Universität Regensburg.

Gräsel, C., Fußangel, K. \& Pröbstel, C. (2006). Lehrkräfte zur Kooperation anregen - eine Aufgabe für Sisyphos? Zeitschrift für Pädagogik, 52(2), 205-219.

Gugerli-Dolder, B., Traugott, E. \& Frischknecht-Tobler, U. (2013). Emotionale Kompetenzen in der Bildung für Nachhaltige Entwicklung, BNE-Konsortium COHEP, Schweizerische Koordinationskonferenz Bildung für eine Nachhaltige Entwicklung. Zürich: o.V.

Hampl, U. (2000). Außerschulische Lernorte im Biologieunterricht der Realschule. Untersuchungen zu kognitiven und affektiven Aspekten am Beispiel des außerschulischen Lernortes „Lehrbienenstand“. Herdecke: GCA.

Harenberg, D. \& de Haan, G. (1999). Bildung für eine nachhaltige Entwicklung. Gutachten zum Programm, FU Berlin. In Bund-Länder-Kommission (Hrsg.), Materialien zur Bildungsplanung und Forschungsförderung (H. 72). Bonn: BLK.

Haubenhofer, D., Demattio, L. \& Geber, S. (2012). Wirkung und Nutzen von Green Care. Eine Recherche und Analyse fachbezogener Artikel. Wien: Landwirtschaftskammer Wien.

Haubenhofer, D. \& Wolf, R. (2015). Lernen und Lehren im Garten. Analyse über Ursprung, Definition, Abgrenzung und Wirkung von Gartenpädagogik. Wien: Hochschule für Agrar- und Umweltpädagogik.

Helmcke, M. (2008). Handbuch für Netzwerk-und Kooperationsmanagement. Bielefeld: USP Publishing.

Künzli David, C. \& Bertschy, F. (2008). Didaktisches Konzept Bildung für eine nachhaltige Entwicklung. Bern: Interfakultäre Koordinationsstelle für Allgemeine Ökologie an der Uni Bern.

Messmer, K., Niederhäuser, R. von, Rempfler, A. \& Wilhelm, M. (2011). Außerschulische Lernorte - Positionen aus Geographie, Geschichte und Naturwissenschaften. Münster: LIT.

Niedersächsisches Kultusministerium (2019). Außerschulische Lernstandorte in einer Bildung für nachhaltige Entwicklung (BNE). Zugriff am 05.11.2019 https://www. mk.niedersachsen.de/startseite/schule/schulerinnen_und_schuler_eltern/bildung_ fur_nachhaltige_entwicklung_bne/ausserschulische_lernstandorte_bne/regionaleumweltbildungszentren-ruz-6341.html
Pauser, N. (2009). Exklusive Behinderungen! Inklusive Beratung? In S. Abdul-Hussain (Hrsg.), Diversity in Supervision, Coaching und Beratung (S. 141-171). Wien: facultas.wuv.

Programm Transfer-21 Koordinierungsstelle Freie Universität Berlin (Hrsg.) (2007). Orientierungshilfe Bildung für nachhaltige Entwicklung in der Sekundarstufe I. Begründungen, Kompetenzen, Lernangebote. Berlin: BLK - Bund-Länder-Kommission. Reuschenbach, M. (2009). Das Potenzial regionaler Geographie zur Förderung einer nachhaltigen Verhaltenskompetenz. In M. Flath, J. Schockemöhle (Hrsg.), Regionales Lernen - Kompetenzen fördern und Partizipation stärken. (S. 25-36) Weingarten: Selbstverl. des Hochschulverbes für Geographie und ihre Didaktik.

Schockemöhle, J. (2009). Außerschulisches regionales Lernen als Bildungsstrategie für eine nachhaltige Entwicklung. Weingarten: Selbstverlag Hochschulverband für Geographie und ihre Didaktik e.V.

Tiller, C. (im Druck). Regionales außerschulisches Lernen im inklusiven Geographieunterricht. Das Potenzial des Bildungskonzeptes „Regionales Lernen 21+“ für inklusive Lernsettings am Beispiel eines Lernangebotes für die Aneignung wirtschaftsgeographischer Lerninhalte im Rahmen einer Betriebserkundung. In B. Baumert \& M. Willen (Hrsg.), Die Werkstatt Inklusion. Qualitätsoffensive Lehrerbildung an der Universität Vechta (S. 125-138). Münster: Waxmann.

Wappel, M. \& Kropf, S. (2018). Gartenpädagogik. Tulln an der Donau: Verein „Natur im Garten". Zugriff am 12.11.2019 https://www.bildungslandkarte.at/uploads/ tx_hetobildungslandkarte/716_projtext_2.pdf

\section{Dr. Gabriele Diersen}

ist wissenschaftliche Mitarbeiterin an der Universität Vechta in der Fakultät II im Fach Geographie. Sie ist seit 2011 geschäftsführende Leiterin des Kompetenzzentrums Regionales Lernen.

\section{Dr. Lara Paschold}

ist Hochschulprofessorin an der Hochschule für Agrar- und Umweltpädagogik Wien im Institut für Beratung, Entwicklungsmanagement und E-Learning/E-Didaktik.

\section{UNSERE BUCHEMPFEHLUNG}

Ursula Bertels, Claudia Bußmann

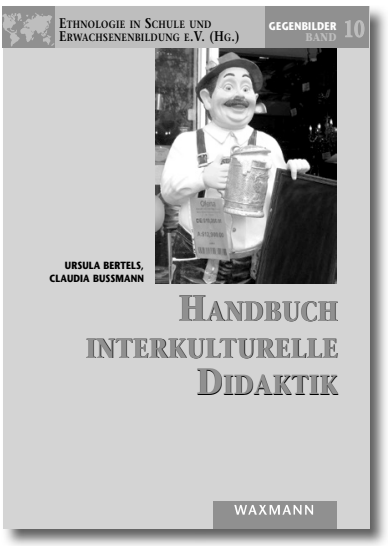

Gegenbilder, Band 10, 2020, 2. korrigierte Auflage, 236 Seiten, br., 24,90€ ISBN 978-3-8309-4212-2 E-Book: $21,99 €$ ISBN 978-3-8309-9212-7

\section{Handbuch interkulturelle Didaktik}

Nur durch interkulturelles und globales Lernen ist es möglich, Kindern und Jugendlichen interkulturelle Kompetenz zu vermitteln und sie damit auf ein Leben in einer multikulturellen Gesellschaft auf globaler und lokaler Ebene vorzubereiten. Ziel des Handbuchs ist es, die theoretischen Hintergründe des interkulturellen und globalen Lernens sowie die praktischen Umsetzungsmöglichkeiten aufzuzeigen. Obwohl der Schwerpunkt des Handbuchs auf der Schule liegt, lassen sich die vorgestellten Methoden und Materialien in angepasster Form auch in der Erwachsenenbildung einsetzen. 